\title{
Local and sex-specific biases in crossover vs. noncrossover outcomes at meiotic recombination hot spots in mice
}

\author{
Esther de Boer, ${ }^{1,4}$ Maria Jasin, ${ }^{2}$ and Scott Keeney ${ }^{1,3}$ \\ ${ }^{1}$ Molecular Biology Program, ${ }^{2}$ Developmental Biology Program, ${ }^{3}$ Howard Hughes Medical Institute, Memorial Sloan Kettering \\ Cancer Center, New York, New York 10065, USA
}

\begin{abstract}
Meiotic recombination initiated by programmed double-strand breaks (DSBs) yields two types of interhomolog recombination products, crossovers and noncrossovers, but what determines whether a DSB will yield a crossover or noncrossover is not understood. In this study, we analyzed the influence of sex and chromosomal location on mammalian recombination outcomes by constructing fine-scale recombination maps in both males and females at two mouse hot spots located in different regions of the same chromosome. These include the most comprehensive maps of recombination hot spots in oocytes to date. One hot spot, located centrally on chromosome 1, behaved similarly in male and female meiosis: Crossovers and noncrossovers formed at comparable levels and ratios in both sexes. In contrast, at a distal hot spot, crossovers were recovered only in males even though noncrossovers were obtained at similar frequencies in both sexes. These findings reveal an example of extreme sex-specific bias in recombination outcome. We further found that estimates of relative DSB levels are surprisingly poor predictors of relative crossover frequencies between hot spots in males. Our results demonstrate that the outcome of mammalian meiotic recombination can be biased, that this bias can vary depending on location and cellular context, and that DSB frequency is not the only determinant of crossover frequency.
\end{abstract}

[Keywords: meiosis; recombination; crossing over; gene conversion; Spol1; hot spot]

Supplemental material is available for this article.

Received May 15, 2015; revised version accepted July 21, 2015.

During meiosis, homologous chromosomes (homologs) pair and undergo reciprocal DNA exchanges (crossovers), which are required for proper chromosome segregation and promote genetic diversity. In many eukaryotes, including mammals, both homolog recognition and crossover formation involve homologous recombination initiated by SPO11-generated DNA double-strand breaks (DSBs) (Lam and Keeney 2014). Recombination at multiple chromosomal positions supports homolog pairing, but only a subset of DSBs becomes interhomolog crossovers; the rest give rise to interhomolog noncrossovers or genetically silent sister chromatid recombination (Schwacha and Kleckner 1994; Allers and Lichten 2001; Hunter and Kleckner 2001; Goldfarb and Lichten 2010). Studies in the budding yeast Saccharomyces cerevisiae indicate that the two types of interhomolog recombination products arise through distinct pathways (for review, see Youds and Boulton 2011). Most noncrossovers do not appear to involve Holliday junction intermediates but in-

\footnotetext{
${ }^{4}$ Present address: Plant Development and (Epi)Genetics, Swammerdam Institute of Life Sciences, University of Amsterdam, 1098XH Amsterdam, The Netherlands

Corresponding authors: s-keeney@ski.mskcc.org, m-jasin@ski.mskcc.org Articlue published online ahead of print. Aricle and publication date are online at http://www.genesdev.org/cgi/doi/10.1101/gad.265561.115.
}

stead are thought to arise through synthesis-dependent strand annealing. In contrast, most crossovers are formed by resolution of double Holliday junction intermediates through the canonical DSB repair pathway and require a group of proteins collectively known as the ZMM proteins (Zip1, Zip2, Zip3, Mer3, and Msh4/Msh5) as well as the MutL homologs Mlh1 and Mlh3 for their formation. A subset of crossovers is formed by an alternative pathway involving Holliday junction resolution by distinct resolvases, including Mus81-Mms4 or Yen1. Studies in other organisms indicate that the existence of distinct pathways for forming crossovers versus noncrossovers is a conserved feature of meiosis (e.g., see Cole et al. 2014), although detailed operation of these pathways often differs between taxa (Youds and Boulton 2011).

Recombination is regulated to ensure that each chromosome receives at least one crossover and that multiple crossovers on the same chromosome (if they occur) tend to be widely and evenly spaced (Jones and Franklin

(C) 2015 de Boer et al. This article is distributed exclusively by Cold Spring Harbor Laboratory Press for the first six months after the full-issue publication date (see http://genesdev.cshlp.org/site/misc/terms.xhtml). After six months, it is available under a Creative Commons License (Attribution-NonCommercial 4.0 International), as described at http:// creativecommons.org/licenses/by-nc/4.0/. 
2006). Studies in many species have focused on how multiple recombination events on individual chromosomes are regulated with respect to one another (e.g., see de Boer et al. 2006; Libuda et al. 2013), but less attention has been paid to the question of whether recombination plays out similarly at all genomic locations. Cytological data in mice suggest that there are significant differences between large (tens of megabases) chromosomal domains in the likelihood that a DSB will give rise to a crossover versus noncrossover outcome (de Boer et al. 2006), but direct molecular tests of these differences are lacking.

Most crossovers in humans and mice occur in narrow ( 1- to 2-kb-wide) regions termed hot spots, which overlap preferred sites of SPO11 DSBs (Brick et al. 2012; Baudat et al. 2013). Individual crossover hot spots have been extensively characterized by analysis of human and mouse sperm DNA (Jeffreys et al. 2001; Guillon and de Massy 2002; Bois 2007; Cole et al. 2010). Noncrossovers are less well characterized, but, where examined, occur in the same hot spots in which crossovers occur (Jeffreys and May 2004; Guillon et al. 2005; Cole et al. 2010; Sarbajna et al. 2012). While crossovers are readily identified because they exchange large DNA segments, noncrossovers are only detectable if a sequence polymorphism is copied from the intact homolog during DSB repair. Because noncrossover gene conversion tracts are short, detection is highly dependent on the location of polymorphisms relative to DSBs, which differs between hot spots (Guillon et al. 2005; Cole et al. 2010). Not surprisingly, then, the ratios of detectable noncrossovers to crossovers vary widely between hot spots, ranging from a $>10$-fold excess of crossovers to a 10 -fold excess of noncrossovers, the latter of which is close to the genome average estimated by cytology of DSB and crossover markers (Holloway et al. 2006; Baudat and de Massy 2007b; Cole et al. 2010; Sarbajna et al. 2012). Thus, to what degree the variation in crossover:noncrossover ratios is a consequence of technical limitations or instead reflects genuine differences in recombination outcome has usually been unclear. Moreover, a recent study of DSB formation in human males emphasized recombination outcome similarities between genomic locations by focusing on DSB frequency as the principal determinant of crossover frequency (Pratto et al. 2014).

Comparatively little is known about recombination in females. In mouse pedigree studies of recombination on chromosomes 1 and 11, sex-specific crossover hot spots were identified, and some shared hot spots showed male versus female differences in crossover frequencies (Paigen et al. 2008; Billings et al. 2010). Because pedigree analysis only detects crossovers, it is uncertain whether this sexspecific variation in recombination results from differences in DSB formation, recombination outcome, or both. Molecular studies of recombination in females have been limited given the technical challenges of studying recombination in the fetal ovary, where meiosis occurs. Furthermore, oocytes are embedded within somatic tissue rather than being present in a ductal lumen as for sperm, and the number of oocytes recovered from a female is much less than the number of sperm recovered from a male. Thus far, only one mouse hot spot (Psmb9, initially identified as a female-specific hot spot) (Shiroishi et al. 1990) has been directly assayed for crossovers and noncrossovers in both males and females, but available data in females are limited to relatively few recombinant molecules and/or assays of noncrossover gene conversion at just one or a small number of polymorphisms (Guillon et al. 2005; Baudat and de Massy 2007a; Cole et al. 2014).

In this study, we generated comprehensive, high-resolution maps of crossovers and noncrossovers in both males and females at two previously uncharacterized hot spots located in distinct regions on the same chromosome. Examining the same hot spots in both sexes allowed us to analyze sex-specific variation in recombination outcomes free of confounding effects attributable to the positions of scoreable sequence polymorphisms. We also compared relative crossover frequencies with published estimates of relative DSB frequencies. The findings reveal striking sex-specific and local differences between hot spots in the likelihood that a DSB will give rise to a crossover versus noncrossover recombination product.

\section{Results}

\section{Hot spot selection}

To test the hypothesis that there can be regional and sex-specific variation in recombination outcomes, we selected hot spots located in regions displaying different propensities toward crossover formation. The central approximately one-third of mouse chromosome 1 shows a similar crossover preference in males and females, based on relative frequencies of MSH4 foci (a cytological marker of early recombination intermediates) and MLH1 foci (a marker of crossovers) (de Boer et al. 2006). In contrast, males generate crossovers preferentially in centromeredistal subtelomeric regions (Shifman et al. 2006; Paigen et al. 2008). This is not matched by a higher frequency of MSH4 foci, suggesting that male predilection toward subtelomeric crossovers reflects a regional bias in the crossover:noncrossover ratio, not simply more DSBs (de Boer et al. 2006). If so, this bias is at least partially sex-specific (de Boer et al. 2006; Shifman et al. 2006; Paigen et al. 2008).

We identified a hot spot from the central region of chromosome 1 using recombinant inbred (RI) strains. These strains contain patchworks of different genomic segments from two parental inbred strains, the boundaries of which are determined during the generations of inbreeding by crossovers at potential hot spots (Supplemental Fig. S1; Bois 2007). Sixteen candidate hot spots were initially examined, of which one proved appropriate for further analysis because it has a suitable polymorphism density and lacks repeats that would compromise PCR amplification (see the Supplemental Material). This central hot spot, located at $78.59 \mathrm{Mb}$ on chromosome 1 (Fig. 1A), has a polymorphism density of $0.97 \%$ between the C57BL/6J (hereafter, B6) and A/J haplotypes (30 polymorphisms across 3094 base pairs [bp]) (Supplemental Table $\mathrm{S} 1)$. A second hot spot, at $185.27 \mathrm{Mb}$ on chromosome 1 
A

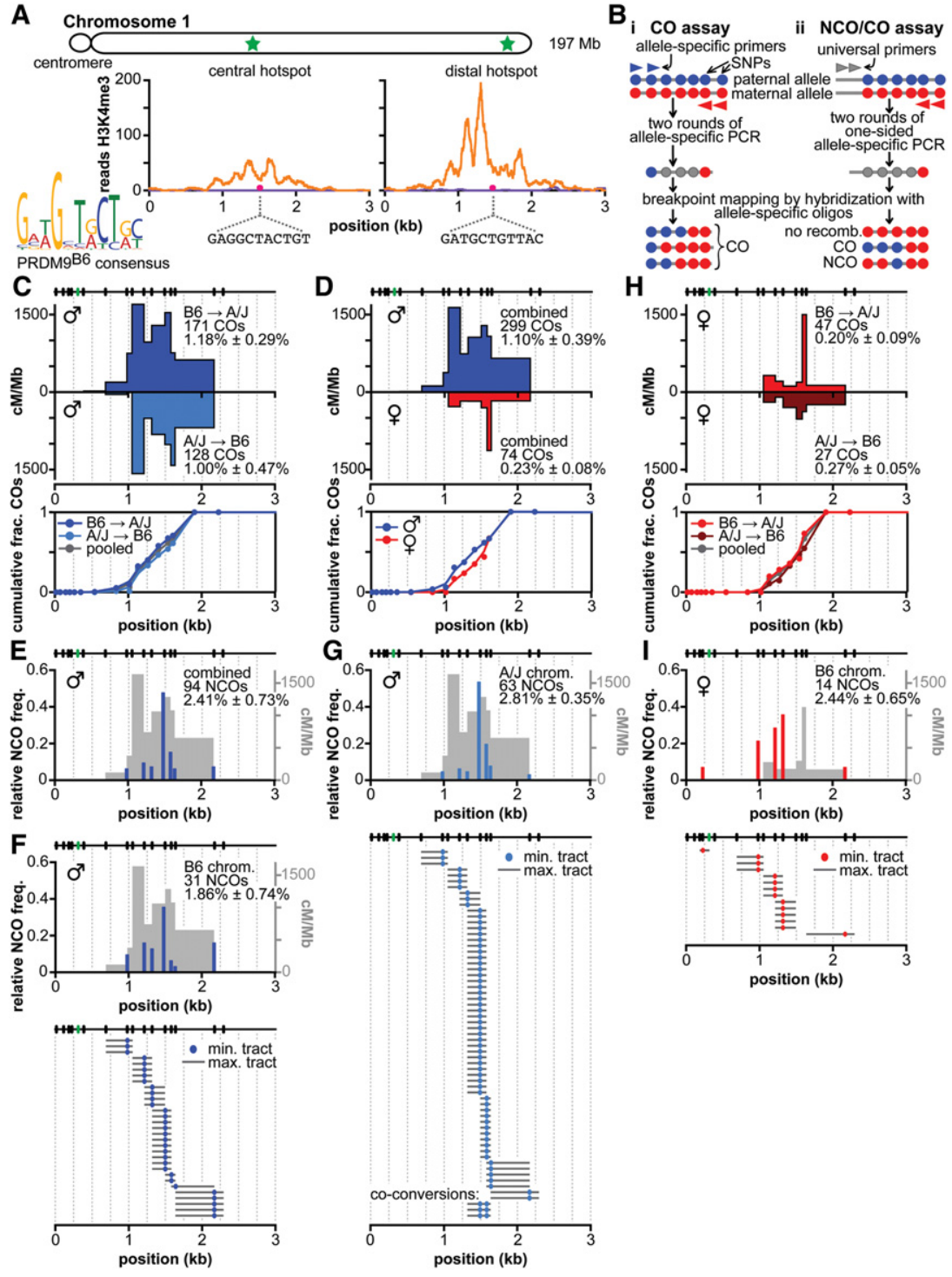

Figure 1. Recombination at the central hot spot in males and females. (A) Overview of recombination hot spots in this study. (Top) Schematic of mouse chromosome 1, with hot spot positions indicated as green stars. The central hot spot is located between base pairs $78,589,305$ and $78,592,399$. The distal hot spot is located between base pairs $185,265,469$ and 185,269,517 (build 38 for both). (Bottom) Both hot spots show H3 Lys4 trimethylation (H3K4me3) ChIP-seq (chromatin immunoprecipitation [ChIP] combined with deep sequencing) signals (Baker et al. 2014) in testes of mice expressing the B6 version of PRDM9 (orange trace) but not the PRDM9 version found in the CAST/EiJ strain (purple trace, which mostly overlaps the horizontal axis). (Bottom left) The 11-bp motif predicted to bind B6 (and A/ J)-encoded PRDM9 (J Lange and S. Tischfield, pers. comm.) is found in both hot spots (pink dots) and is identical in the B6 and A/J strains for each hot spot. The depicted regions in these and all subsequent hot spot graphs are $78,589,447-78,592,447$ for the central hot spot and $185,265,656-185,268,656$ for the distal hot spot. (B) Assays to amplify and identify crossovers and noncrossovers. Filled circles represent sequence polymorphisms (red or blue for the two parental genotypes and gray for amplified but not-yet-defined internal polymorphisms), and arrowheads represent PCR primers (red or blue for allele-specific primers and gray for universal). (Panel i) In the crossover (CO) assay, two sequential rounds of allelespecific PCR selectively amplify recombinant DNA molecules from small pools of sperm or oocyte DNA from an F1 hybrid animal. Recombination frequencies are estimated from the observed fraction of pools that yield amplification products. Next, internal polymorphisms in each amplified recombinant DNA molecule are genotyped by hybridization with allele-specific oligonucleotides (ASOs) to map the location of the crossover breakpoint. (Panel

ii) In the noncrossover/crossover $(\mathrm{NCO} / \mathrm{CO})$ assay, smaller pools of sperm or oocyte DNA are amplified using nested primers that are specific for one haplotype in combination with nested universal primers. In contrast to the crossover assay, both nonrecombinant and recombinant DNA molecules are amplified, with the majority $(>95 \%)$ being nonrecombinants. Subsequent hybridization of amplification products to ASOs that are specific for alleles from the nonselected haplotype identifies pools containing crossovers and noncrossovers. $(C)$ Crossovers in males. (Top graph) Crossover breakpoint maps are shown for crossover molecules amplified with allele-specific primers in the B6-to-A/J orientation (dark blue) or the A/J-to-B6 orientation (light blue). Cumulative crossover distributions are shown below. Tested polymorphisms are indicated as ticks at the top. Multiple polymorphisms contained within a single ASO are indicated as a single green tick. Numbers of observed crossovers and Poisson-corrected crossover frequencies $( \pm S D)$ are indicated. $(D)$ Similar distributions of crossover breakpoints in males and females. Data from both orientations of the allele-specific PCR were pooled separately for males (blue) and females (red). (E) Total noncrossovers in males. Total relative noncrossover frequencies from all four orientations of the PCRs (normalized for co-conversions) at the tested polymorphisms are shown as blue bars. The crossover breakpoint map in males is shown for comparison (light gray). Number of total observed noncrossovers and Poisson-corrected total noncrossover frequency $( \pm S D)$ are indicated. $(F, G)$ Noncrossovers on the $\mathrm{B} 6(F)$ and $\mathrm{A} / \mathrm{J}(G)$ chromosomes in males. (Top) Relative noncrossover frequencies (normalized for coconversions) on the $\mathrm{B} 6(F)$ and $\mathrm{A} / \mathrm{J}(G)$ chromosomes at the tested polymorphisms are shown as blue bars. The crossover breakpoint map in males is shown for comparison (light gray). Number of observed noncrossovers and Poisson-corrected noncrossover frequency $( \pm \mathrm{SD})$ are indicated. (Bottom) Noncrossover gene conversion tracts on the B6 $(F)$ and A/J $(G)$ chromosomes. $(H)$ Crossovers in females. Crossover breakpoint maps $(t o p$ graph) and cumulative crossover distributions (bottom graph) are shown for the B6-to-A/J orientation (light red) and the A/J-to-B6 orientation (dark red) of allele-specific PCR. (I) Noncrossovers in females. Relative noncrossover frequencies and noncrossover gene conversion tracts from PCR in the universal-to-B6 orientation are presented as for males. 
(Fig. 1A, "distal hot spot"), was selected from a whole-genome map of recombination initiation sites identified by deep sequencing of ssDNA bound by the RAD51 or DMC1 strand exchange protein (Smagulova et al. 2011). The polymorphism density between B6 and A/J haplotypes at this hot spot is $0.89 \%$ (36 polymorphisms across 4048 bp) (Supplemental Table S2).

A principal determinant of DSB hot spot location is the meiosis-specific PRDM9 protein, which contains a histone methyltransferase domain and a DNA-binding domain comprising an array of $\mathrm{Zn}$ finger modules whose DNA-binding specificity evolves rapidly (Baudat et al. 2010; Myers et al. 2010; Grey et al. 2011; Brick et al. 2012). Both the central and distal hot spots contain a match in both the B6 and A/J strains to the motif predicted to bind the B6-encoded PRDM9 (A/J has the same Prdm9 allele), and PRDM9-dependent histone H3 Lys4 trimethylation $(\mathrm{H} 3 \mathrm{~K} 4 \mathrm{me} 3)$ is present at both hot spots as determined by chromatin immunoprecipitation (Fig. 1A; Baker et al. 2014). Thus, both hot spots are predicted to be active sites for DSB formation in $\mathrm{B} 6 \times \mathrm{A} / \mathrm{J}$ F1 hybrids.

\section{Recombination outcomes at the central hot spot in males}

Crossovers We examined crossing over in the central hot spot in males by allele-specific PCR on sperm DNA from B6 $\times$ A/J F1 hybrids (Fig. 1B, panel i). Crossover molecules were selectively amplified from pools of sperm DNA by two rounds of PCR with nested forward primers specific for one parental haplotype combined with nested reverse primers specific for the other parental haplotype (Jeffreys and May 2003). Crossover breakpoints in the amplified recombinant molecules were subsequently mapped by allele-specific hybridization at 18 polymorphisms across the hot spot. We retrieved 299 crossover molecules from a total input of $\sim 32,000$ haploid genome equivalents, for a Poisson-corrected overall frequency of $1.10 \%$ per haploid genome (Table 1). No crossovers were detected in somatic DNA controls (frequency <0.004\%). Crossovers showed similar frequencies and breakpoint distributions for both orientations of allele-specific primers (i.e., B6 forward primers plus A/J reverse or the converse) (Fig. 1C). The similar breakpoint distributions imply that recombination initiates at a similar frequency on both haplotypes in this F1 hybrid strain, as inferred previously at other hot spots (Jeffreys and Neumann 2002; Cole et al. 2010). Combining data from both primer orientations, crossover activity spanned $1.8 \mathrm{~kb}$, with an average of $627 \mathrm{cM} / \mathrm{Mb}$ and peak of $1638 \mathrm{cM} / \mathrm{Mb}$ (Fig. 1D, ơ). These values place this hot spot among the most active hot spots for crossovers characterized in male mice, comparable with Psmb9 (1.1\% crossover frequency and $1300 \mathrm{cM} / \mathrm{Mb}$ peak activity) (Guillon and de Massy 2002).

Noncrossovers To detect noncrossovers, we used nested PCRs that were allele-specific on just one side; i.e., with primers for one of the parental haplotypes opposed to "universal" primers that amplify both haplotypes (Fig. 1B, panel ii; Jeffreys and May 2004). Recombinant DNA molecules amplified from small pools of sperm DNA were detected by hybridization to allele-specific oligonucleotides directed against the nonselected parental haplotype. From all four possible orientations of allele-specific primers, we recovered 94 noncrossovers from a total input of $\sim 4200$ haploid genome equivalents, for a Poisson-corrected overall frequency of $2.4 \%$ per haploid genome (Fig. 1E; Table 1; Supplemental Table S1). Noncrossovers occurred across the same region as crossovers, and the peak of noncrossover activity overlapped the center of the crossover distribution (Fig. 1E-G). The polymorphism showing the highest noncrossover frequency was adjacent to the PRDM9 motif (relative noncrossover frequencies of 0.36 on the B6 chromatid and 0.56 on the A/J chromatid). The minimum gene conversion tract of each noncrossover was measured by considering only the polymorphisms involved plus the segments in between if more than one polymorphism was converted; the

Table 1. Summary of recombination outcomes at the central and distal hot spots

\begin{tabular}{|c|c|c|c|c|}
\hline & \multicolumn{2}{|c|}{$0^{+}$} & \multicolumn{2}{|c|}{ 운 } \\
\hline & Central & Distal & Central & Distal \\
\hline $\begin{array}{l}\text { Crossover frequency from crossover assay }{ }^{\mathrm{a}} \\
\text { (number/total) }^{\mathrm{b}}\end{array}$ & $\begin{array}{c}1.10 \% \pm 0.39 \% \\
(299 / 32,290)\end{array}$ & $\begin{array}{c}0.36 \% \pm 0.03 \% \\
(269 / 93,102)\end{array}$ & $\begin{array}{l}0.23 \% \pm 0.08 \% \\
(74 / 29,438)\end{array}$ & $\begin{array}{l}<0.007 \% \\
(0 / 15,013)\end{array}$ \\
\hline $\begin{array}{l}\text { Crossover frequency from noncrossover/ } \\
\text { crossover assay }^{\mathrm{a}}(\text { number/total })^{\mathrm{b}}\end{array}$ & $\begin{array}{c}1.15 \% \pm 0.31 \% \\
(47 / 4177)\end{array}$ & $\begin{array}{l}0.33 \% \pm 0.12 \% \\
(25 / 8007)\end{array}$ & $\begin{array}{l}0.34 \% \pm 0.24 \% \\
(2 / 584)\end{array}$ & $\begin{array}{l}<0.017 \% \\
(0 / 5994)\end{array}$ \\
\hline $\begin{array}{l}\text { Noncrossover frequency from noncrossover/ } \\
\text { crossover assay }^{\mathrm{a}} \text { (number/total }^{\mathrm{b}}\end{array}$ & $\begin{array}{l}2.41 \% \pm 0.73 \% \\
\quad(94 / 4177)\end{array}$ & $\begin{array}{l}1.53 \% \pm 0.44 \% \\
(115 / 8007)\end{array}$ & $\begin{array}{c}2.44 \% \pm 0.65 \% \\
(14 / 584)\end{array}$ & $\begin{array}{l}2.26 \% \pm 0.80 \% \\
(122 / 5994)\end{array}$ \\
\hline Crossover:noncrossover ${ }^{\mathrm{c}}$ & $1: 4.2$ & $1: 9.3$ & $1: 14.4^{\mathrm{d}}$ & $<1: 266$ \\
\hline SSDS reads ${ }^{\mathrm{e}}$ & 2819 & 9247 & $\mathrm{NA}^{\mathrm{f}}$ & $\mathrm{NA}^{\mathrm{f}}$ \\
\hline
\end{tabular}

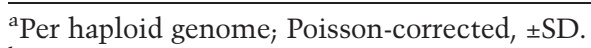

bobserved number of recombinants and total haploid genome equivalents analyzed.

${ }^{\mathrm{c}}$ Ratio calculated from per-meiosis recombination frequencies [(noncrossover $\left.\times 4\right) \div($ crossover $\left.\times 2)\right]$ from the noncrossover/crossover assay.

${ }^{\mathrm{d}}$ Note that this ratio is based on a small number of events.

${ }^{\text {e}}$ From Brick et al. (2012).

f(NA) Data not available. 
maximum conversion tract includes the distance to the nearest flanking polymorphisms that were not converted (Fig. 1F,G). For the combined noncrossovers on both the B6 and A/J chromosomes, the average minimum and maximum conversion tracts were $4 \mathrm{bp}$ and $304 \mathrm{bp}$, respectively. Both the distribution of noncrossovers across the hot spot and the conversion tract lengths are similar to reports for other hot spots (Baudat and de Massy 2007b; Cole et al. 2010). Coconversions were rare: Only three noncrossovers converted more than one polymorphism, all involving the same two polymorphisms near the hot spot center, with minimum and maximum tract lengths of $79 \mathrm{bp}$ and 315 bp, respectively (Fig. 1G). These coconversions were independent events, as they arose from separate sperm pools.

Importantly, this assay also detects crossovers (Fig. 1B, panel ii). We recovered 47 crossover molecules, for a Poisson-corrected overall frequency of $1.15 \%$ (Table 1). Agreement of this value with that from the crossover-specific assay validates direct quantitative comparison between them. Each noncrossover recombination event generates a single recombinant DNA molecule from four chromatids, whereas each crossover event generates two (reciprocal) recombinants (Cole et al. 2014). Thus, the relative numbers of detectable recombinants in the noncrossover/crossover assay translate to a per-meiosis ratio of one crossover to 4.2 noncrossovers (crossovers: $2.30 \%$ of meioses $[2 \times 1.15 \%]$; noncrossovers: $9.64 \%$ of meioses $[4 \times 2.41 \%])$. This is lower than the $\sim 1: 10$ ratio estimated for genome average and observed at the well-characterized A3 hot spot (Baudat and de Massy 2007b; Cole et al. 2010).

\section{Recombination outcomes at the distal hot spot in males}

Crossovers In the crossover-specific assay, we recovered 269 crossover molecules at the distal hot spot from 23,000 haploid genome equivalents of sperm DNA from $\mathrm{B} 6 \times \mathrm{A} / \mathrm{J}$ F1 hybrids, for a Poisson-corrected overall frequency of $0.36 \%$ (Fig. 2A,B; Table 1). Crossover breakpoints showed similar distributions for both PCR orientations (Fig. 2A), again implying equivalent DSB frequencies on both haplotypes. No crossovers were detected in somatic DNA controls (frequency <0.004\%). Breakpoint mapping using hybridization to allele-specific oligonucleotides (ASOs) at 25 polymorphisms across the hot spot indicated that crossovers occurred across a $2.7-\mathrm{kb}$ region, with most breakpoints in the central $1 \mathrm{~kb}$. The hot spot averaged $116 \mathrm{cM} / \mathrm{Mb}$, peaking at $361 \mathrm{cM} / \mathrm{Mb}$ (Fig. 2B, o). Although not as active for crossing over as Psmb9 or the central hot spot, the distal hot spot is very active in males and is similar to $A 3(0.26 \%$ crossover frequency in $\mathrm{B} 6 \times$ DBA F1 hybrids) (Guillon and de Massy 2002; Cole et al. 2010).

Noncrossovers We identified 115 noncrossovers from >8000 haploid genome equivalents of sperm DNA, for a Poisson-corrected frequency of $1.53 \%$ (Fig. 2C-E, ơ; Table 1; Supplemental Table S2). Average minimum and maxi- mum conversion tracts were 2 bp and 294 bp, respectively, comparable with $A 3$ (Cole et al. 2010) and the central hot spot, and coconversions were scarce (Fig. 2D,E). Three noncrossovers were detected that hybridized to two adjacent ASOs of the opposing allele. These coconversions involved the same two polymorphisms $11 \mathrm{bp}$ apart, located on the left flank of the hot spot (maximal tract $483 \mathrm{bp}$ ) (Fig. 2D). Six noncrossovers are presumptive coconversions because they hybridized to ASOs that each contained two single nucleotide polymorphisms (SNPs; polymorphisms 3 and 6 bp apart) (Supplemental Table S2).

The same PCRs yielded 25 crossover molecules (Poisson-corrected frequency of $0.33 \%$ ) (Table 1 ), matching expectation from the crossover-specific assay. The observed per-meiosis ratio is one crossover to 9.3 noncrossovers $[(4 \times 1.53 \%$ noncrossovers $) \div(2 \times 0.33 \%$ crossovers $)]$. This is comparable with the ratio at $A 3$ (Cole et al. 2010). However, the middle of the hot spot contains a 634-bp stretch without polymorphisms between the B6 and A/J haplotypes (Fig. 2; Supplemental Table S2). Thus, it is possible that noncrossovers occur frequently in this interval but escape detection.

\section{DSB levels and crossover frequencies are poorly correlated}

To compare local DSB activity with recombination outcomes, we analyzed published data generated by deep sequencing of ssDNA bound by the DMC1 strand exchange protein in testis extracts from B6 animals (Brick et al. 2012). In this ssDNA sequencing (SSDS) assay, reads mapping to the forward and reverse strands represent DSB resection tracts to a DSB hot spot's left or right side, respectively. Thus, although precise DSB distributions cannot be gleaned from these data, hot spot midpoints can be inferred to lie between the forward and reverse strand accumulations. Furthermore, total SSDS read count at a hot spot is expected to be proportional to DSB frequency (Pratto et al. 2014).

As expected, each hot spot displayed a cluster of SSDS reads centered on the midpoint of crossover activity (Fig. $3 \mathrm{~A})$. However, the relative SSDS read count correlated poorly with the relative crossover frequency: Compared with the distal hot spot, the central hot spot had less than one-third the frequency of SSDS reads but a 3.5fold higher crossover frequency (Fig. 3A; Table 1). The high DSB activity at the distal hot spot reinforces our suspicion that the actual noncrossover frequency at this hot spot is likely higher than we are able to detect due to the low polymorphism density in the hot spot center. More importantly, these findings imply that a higher DSB frequency does not necessarily translate into a higher crossover frequency.

To evaluate the generality of these findings, we compared SSDS read counts with published crossover frequencies determined by sperm typing or pedigree analysis at $A 3$ and other hot spots active in the B6 strain (Fig. 3B). Although there was a positive correlation between SSDS counts and crossover frequency, the strength of the correlation was modest, with variation in the SSDS 
de Boer et al.
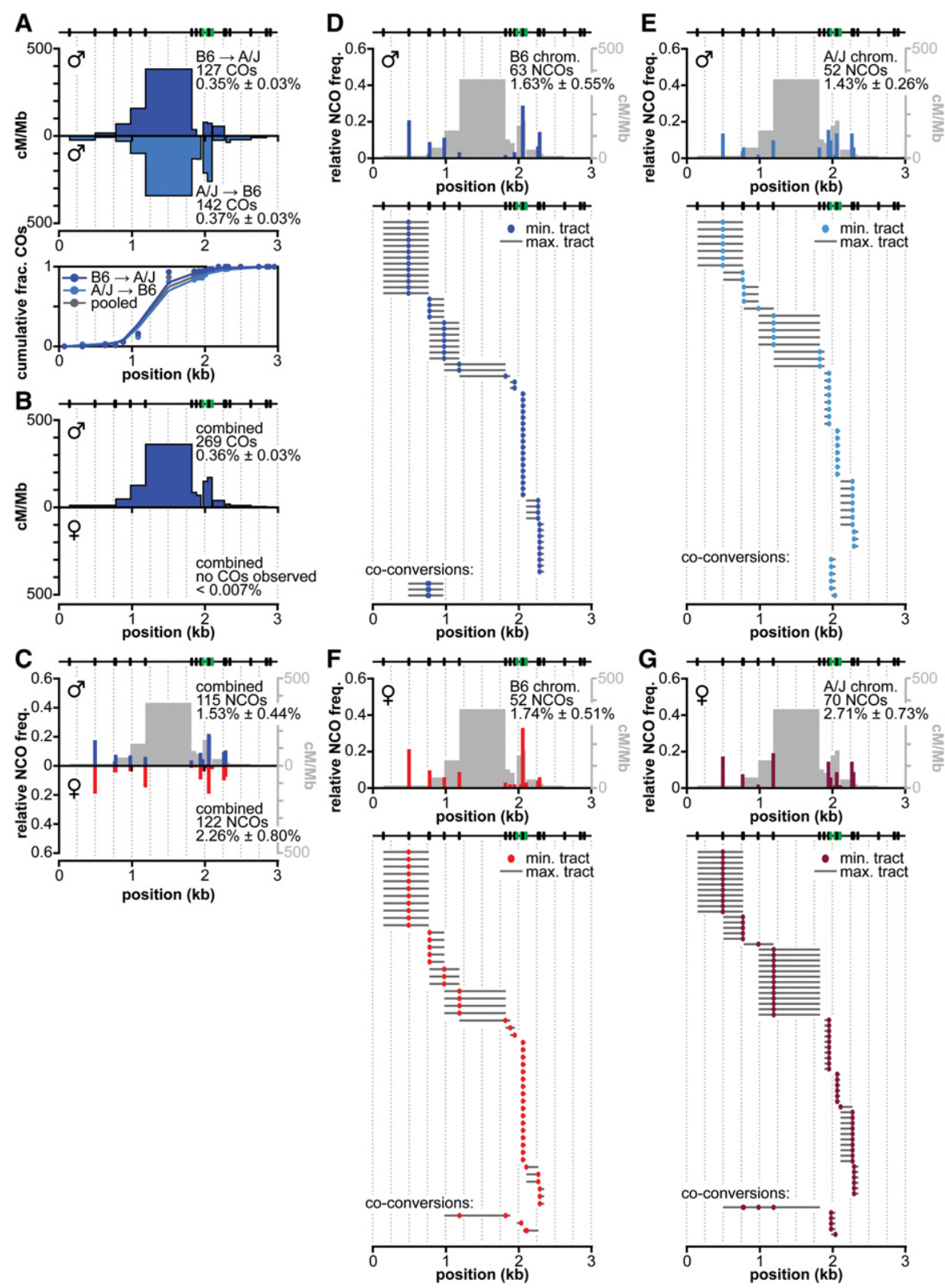

Figure 2. Recombination at the distal hot spot in males and females. (A) Similar distributions of crossover breakpoints in males in both orientations. Crossover breakpoint maps and cumulative crossover distributions are shown as in Figure $1 \mathrm{C}$. $(B)$ Difference in crossover formation between males and females. Data from both orientations of the allele-specific PCR were pooled for males and females, presented as in Figure 1D. No crossover molecules were recovered from oocyte DNA samples. (C) Total noncrossovers in males and females, presented as in Figure 1E. $(D-G)$. Relative noncrossover frequencies and noncrossover gene conversion tract distributions in males $(D, E)$ and females $(F, G)$ on the $\mathrm{B} 6(D, F)$ and $\mathrm{A} / \mathrm{J}(E, G)$ chromosomes, presented as in Figure 1F.

counts explaining less than half the variation in the crossover data $\left(R^{2}=0.44\right)$. Considering subsets of these hot spots is particularly revealing. For example, the hot spots highlighted by the box in Figure 3B are within a factor of two of each other for SSDS signal but cover a 38 -fold range in crossover activity. A similarly poor correlation was seen for data for human hot spots, but emphasis was placed on the existence of a correlation, not deviations of individual hot spots from the trend (Pratto et al. 2014). These findings strongly support the conclusion that the per-DSB crossover frequency can vary substantially between hot spots.

The central hot spot is active in females, generating both crossovers and noncrossovers

To analyze recombination in females, we carried out the crossover and noncrossover/crossover assays on DNA extracted from ovaries of newborn $\mathrm{B} 6 \times \mathrm{A} / \mathrm{J}$ F1 hybrids. At this age, ovaries contain oocytes that are mainly in the 

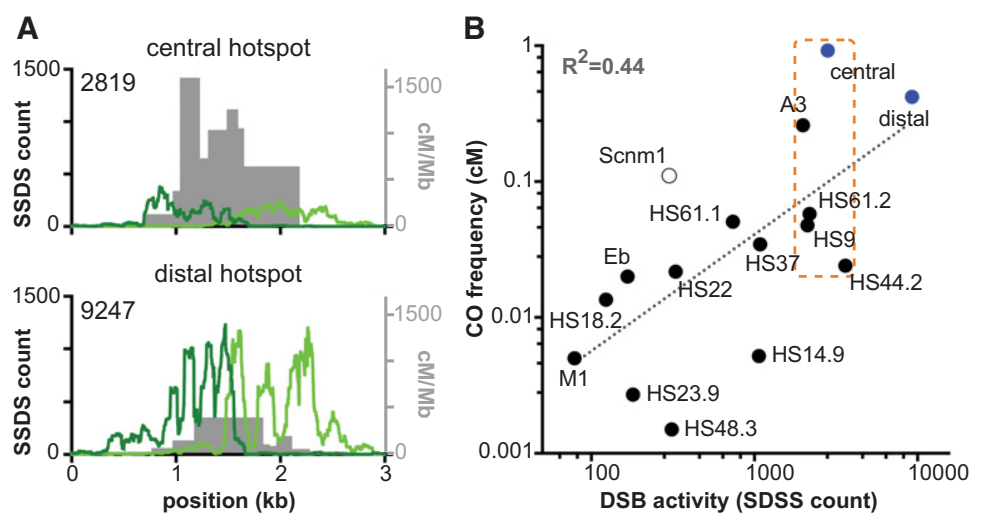

Figure 3. Estimates of relative DSB activity are an unreliable predictor of crossover frequency. $(A)$ Comparison of SSDS reads with crossover distributions at the central and distal hot spots. Crossover breakpoint maps from spermatocytes (pooled from both orientations of allelespecific PCR) are shown in gray. Forward strand SSDS reads are shown in dark green, and reverse strand reads are in light green; total read counts (a measure of DSB activity) are indicated (data from Brick et al. 2012). The midpoint between accumulations of the forward and reverse strand reads marks the hot spot center. Note that A/J and B6 share the same Prdm 9 allele, and the symmetry of crossover maps (Figs. 1C, 2A) indicates that recombination initiation occurs at comparable frequencies on both haplotypes. Thus, the SSDS and crossover maps are directly comparable even though they were generated from animals of different strain backgrounds (pure B6 for SSDS vs. A/J $\times$ B6 F1 hybrids for crossovers). Note that the $Y$-axis scales are the same for both hot spots. (B) Comparison of SSDS read counts and crossover frequencies at published mouse hot spots. SSDS data are from Brick et al. (2012). Filled blue circles are the hot spots from this study, filled black circles denote published hot spots assayed by allelespecific PCR of sperm DNA, and the open circle denotes a published hot spot assayed for crossing over by pedigree analysis (see Supplemental Table S5 for details). The dotted line is a least squares regression line fitted to the log transformed data. The orange box marks a group of hot spots that are within a factor of two of each other for SSDS signal but cover a much wider range in crossover activity. Note that different studies used different methods to correct recombination assays for amplifications efficiencies, so measured crossover frequencies may be underestimated to different degrees for specific hot spots (see the Materials and Methods for further details).

late prophase stages of diplonema and/or dictyate arrest (Dietrich and Mulder 1983; McClellan et al. 2003). Analysis of the kinetics of interhomolog recombination in male mouse meiosis found that most crossovers and noncrossovers were formed by late pachynema, with little to no difference in timing between the two products (Guillon et al. 2005). Assuming comparable kinetics for female meiosis, oocytes from ovaries of newborns are expected to have largely completed meiotic recombination (Guillon et al. 2005; Baudat and de Massy 2007a). Thus, although we analyzed recombination in females at an earlier stage than in males, it is unlikely that this difference would lead to underrepresentation of one or both recombination products.

Because most cells in ovary samples are somatic, we enriched for oocytes by disrupting the ovaries in the presence of collagenase and DNase I followed by several wash steps to selectively lyse and deplete somatic cells (Eppig and Schroeder 1989; de Boer et al. 2013). We determined the fraction of oocytes in the enriched cell suspensions using immunocytology and corrected recombination frequencies accordingly (Baudat and de Massy 2007a, 2009; de Boer et al. 2013).

Crossovers Allele-specific PCR in both orientations yielded 74 crossovers from a total input of $\sim 29,000$ haploid genome equivalents from oocytes, for a Poisson-corrected overall crossover frequency of $0.23 \%$ (Table 1 ). No crossovers were detected in somatic controls (frequency< $0.002 \%$ ). As in males, crossover breakpoints showed similar distributions for both orientations of allele-specific primers (Fig. 1H), indicating no preference for either haplotype in recombination initiation (Jeffreys and Neumann 2002; Cole et al. 2010). Crossover breakpoints showed a distribution similar to that in males (Fig. 1D). The average activity was $244 \mathrm{cM} / \mathrm{Mb}$, with a maximum of $1130 \mathrm{cM} /$ $\mathrm{Mb}$. The lower peak activity and overall frequency in fe- males than in males may reflect a difference in crossover activity between the sexes. Alternatively, the observed difference could reflect different levels of precision in estimating absolute frequencies of recombinant DNA molecules from ovary versus sperm samples. Nonetheless, this hot spot is highly active in females, with an activity $>400$-fold greater than the genome average of $0.55 \mathrm{cM} / \mathrm{Mb}$.

Noncrossovers From PCR in the universal-to-B6 orientation, we recovered 14 noncrossovers from an input of 584 haploid genome equivalents from oocytes, for a Poisson-corrected frequency of $2.4 \%$ (Fig. 1I; Table 1; Supplemental Table S1), a frequency comparable with that of males. The average minimal and maximal conversion tracts were also similar to males ( $1 \mathrm{bp}$ and $304 \mathrm{bp}$, respectively). No coconversions were found among the 14 noncrossovers, agreeing with the low number in males. The majority of noncrossovers was within the central $2 \mathrm{~kb}$ of the hot spot, similar to that observed in males. We also retrieved two crossover molecules from the same assay $(0.34 \%)$, consistent with the crossover assay. The observed per-meiosis ratio is higher than in males (one crossover to 14 noncrossovers), but this difference should be viewed with caution, as the small number of recombinants analyzed in females renders this estimate less precise. Overall, however, we can conclude that this hot spot behaves similarly in males and females.

\section{Crossovers are not detected at the distal hot spot in females}

Crossovers In stark contrast to males, females displayed no detectable crossover activity at the distal hot spot. No crossovers were recovered from a total input of $\sim 15,000$ haploid genome equivalents from oocytes by allele-specific PCR, for an overall crossover frequency of 
$<0.007 \%$ (Fig 2B, o; Table 1). Crossovers were also not detected in somatic controls (frequency $<0.004 \%$ ). Because we could detect crossovers at the central hot spot in females, the absence of crossovers at the distal hot spot cannot be ascribed to technical difficulty in detecting recombinants in oocytes but must instead reflect a sex-specific difference in the behavior of this hot spot.

Noncrossovers To determine whether the absence of crossovers reflects an absence of recombination and thus likely DSBs, we analyzed noncrossover formation in oocytes using all four PCR orientations. From a total input of $\sim 6000$ haploid genome equivalents, we recovered 122 noncrossovers, for a Poisson-corrected overall frequency of $2.3 \%$ (Fig. 2C, \&; Table 1; Supplemental Table S2). This value is comparable with the total recombination frequency in males, and both the spatial distribution and average conversion tract lengths of noncrossovers (minimal, 10 bp; maximal, $345 \mathrm{bp}$ ) were similar in both sexes (Fig. 2C,F,G). Again, coconversions were rare: Three unambiguously detected coconversions included more than one polymorphism (Fig. 2F,G), and seven noncrossovers involved presumptive conversion of more than one polymorphism contained within a single central ASO (Supplemental Table S2). Most coconversions were located at the right flank of the hot spot and were comparable with those found in males in that they involved two closely spaced polymorphisms (minimum conversion tract length of $5 \mathrm{bp}$; maximum conversion tract length of 208 bp). Two coconversions had substantially longer conversion tracts than any events observed in males. One involved four polymorphisms, spanning at least 420 bp (maximum tract length, $1324 \mathrm{bp}$ ); another involved two polymorphisms spanning $634 \mathrm{bp}$ in the center of the hot spot (maximum tract length, $903 \mathrm{bp}$ ). As in the crossover-specific assay, no crossovers were recovered from these PCRs (crossover frequency < $0.017 \%$ ) (Table 1 ).

Thus, this hot spot is highly active for recombination initiation in female meiosis despite the lack of detectable crossovers. These data strongly indicate that the sex-specific differences in crossover activity principally reflect differences in the recombination outcome between males and females rather than a difference in DSB number per se.

\section{Biased gene conversion in noncrossovers}

The large number of noncrossovers identified at the distal hot spot in both males and females provided an opportunity to directly examine gene conversion bias at individual polymorphisms. Gene conversion is biased in favor of transmission of GC alleles in many eukaryotes (Duret and Galtier 2009). Such a bias has been observed directly in noncrossover (Odenthal-Hesse et al. 2014) and crossover (Arbeithuber et al. 2015) recombination products in human sperm and can explain patterns of GC content enrichment at mouse hot spots (Clement and Arndt 2013). Accordingly, we found that, in males, noncrossovers frequently showed significant bias, resulting in more conversion to GC than to AT (Supplemental Table S2). Of the eight GC/AT polymorphisms, six showed bias toward
GC conversion. Normalizing for the number of chromosomes analyzed for noncrossovers, overall, there were 64 conversions to GC but only 33 conversions to AT $(P=$ 0.0022 , binomial test). Bias was observed throughout the hot spot, including at polymorphisms located $1 \mathrm{~kb}$ from the hot spot center. The more limited analysis at the central hot spot also showed a skewing toward GC conversion (29 conversions to GC and 15 conversions to AT; $P=$ 0.049, binominal test) (Supplemental Table S1).

In females, gene conversion bias was not as clear cut, consistent with the suggestion that biased gene conversion is more prominent in males in humans than in females (Duret and Galtier 2009). Four of the six GC/AT polymorphisms that showed bias in males were also skewed toward GC conversion in females (Supplemental Table S2). However, overall, the bias was not significant $(P=0.4926$, binomial test; 56 conversions to GC and only 48 conversions to AT).

\section{Discussion}

Because of the uncertainties in precisely determining noncrossover frequencies, it has been difficult to know whether previously observed differences in crossover:noncrossover ratios between different hot spots (Holloway et al. 2006; Baudat and de Massy 2007b; Cole et al. 2010) reflect genuine differences in the crossover versus noncrossover decision or are merely a result of shortcomings in noncrossover detection. In this study, we provide strong evidence for substantial variation in mouse interhomolog recombination outcomes (crossover vs. noncrossover). This lack of uniformity has important implications for understanding crossover control in complex genomes.

\section{Intrinsic differences between hot spots in the likelihood that a DSB will give rise to a crossover}

In females, noncrossovers were detected at high frequency at both the central and distal hot spots, thus indicating substantial DSB formation at both hot spots, but crossovers, which can unambiguously be determined, were observed in only the central hot spot. Thus, irrespective of the exact frequency of noncrossovers, the central hot spot in females is markedly more biased toward crossover formation than the distal hot spot. In males, we found an analogous difference between the two hot spots by comparing relative crossover frequencies with inferred DSB levels: A 3.5-fold higher crossover frequency was observed at the central hot spot, yet it has one-third the number of SSDS reads.

Analysis of published data for several hot spots located across the genome also shows that SSDS reads as a measure of relative DSB levels are poor predictors of relative crossover frequencies, further supporting the conclusion that there are intrinsic differences between hot spots in the likelihood that a DSB will give rise to a crossover. A similarly modest correlation is obtained with an alternative method of estimating relative DSB levels, SPO11 oligonucleotide sequencing, suggesting that this pattern is not simply due to uncertainty in estimating relative 
DSB frequency from SSDS reads $\left(R^{2}=0.35\right)$ (J Lange, $M$ Jasin, and S Keeney, unpubl.).

In principle, the strong bias against interhomolog crossovers at the distal hot spot in females could be due to factors acting over a large chromosomal domain. For example, subtelomeric and/or centromeric regions in many species tend to have lower levels of crossover formation, with suppression of DSBs, changes in the crossover:noncrossover ratio, or increased sister chromatid recombination as suggested causes (Drouaud et al. 2006; Chen et al. 2008; Mancera et al. 2008). However, the distal hot spot is located at some distance $(\sim 10 \mathrm{Mb})$ from the telomere, and both male and female crossover hot spots have been mapped even closer to the telomere on the same chromosome (Paigen et al. 2008) such that a largescale telomere effect is unlikely to be the sole reason for bias against crossing over at the distal hot spot. Instead, we speculate that factors operating more locally may play an important role. For example, whereas the central hot spot is intergenic, the distal hot spot is located within the Rab3gap2 gene, with the center of the hot spot overlapping an exon (Supplemental Tables S1, S2).

\section{Modulation of the crossover:noncrossover decision in mice}

In males, the crossover:noncrossover ratios are $\sim 1: 4$ at the central hot spot versus $\sim 1: 9$ at the distal hot spot. The short gene conversion tracts for most noncrossovers makes their detection highly dependent on the distribution of polymorphisms relative to DSBs (Cole et al. 2010). For the distal hot spot, the longest stretch without any polymorphisms (634 bp) is located at the center of the hot spot, which is where the peaks of crossover and noncrossover activity for most hot spots tend to overlap (Baudat and de Massy 2007b; Cole et al. 2010). In contrast, polymorphisms in the central hot spot are distributed more evenly, including at the hot spot center, where noncrossover activity is highest. Thus, although it is likely that noncrossovers are underestimated at both hot spots, we infer that the noncrossover frequency is underestimated to a greater degree at the distal hot spot. Even so, the strong bias toward noncrossover formation at this hot spot in males is much less extreme than that in females. While it remains possible that some DSBs in both hot spots are repaired by sister chromatid recombination, as is known to occur in yeast (Schwacha and Kleckner 1994; Goldfarb and Lichten 2010; Hyppa and Smith 2010), these results provide clear evidence for crossover control at the level of the crossover:noncrossover decision.

Regional differences in recombination outcomes have been observed in the budding yeast $S$. cerevisiae by whole-genome analysis (Mancera et al. 2008), but this study did not control for the density of sequence polymorphisms in hot spots, so differences in noncrossover detection may underlie some of the observed variation in recombination outcome (S Keeney, unpubl.). A separate study found that crossovers but not noncrossovers were substantially lower within $20 \mathrm{~kb}$ of chromosome ends compared with genome average (Chen et al. 2008), also suggestive of regional variation in recombination outcome, with recombination near telomeres relatively biased toward noncrossovers (Chen et al. 2008). However, nonallelic homologous recombination between dispersed repetitive sequences is known to exchange ends of different chromosomes at appreciable frequencies (Louis and Haber 1990; Louis et al. 1994), so these subtelomeric regions may be highly variable in yeast. If so, at least some of the observed reduction in crossover frequency may reflect large-scale structural differences between the ends of homologous chromosomes in the hybrid yeast strains analyzed. More recently, Borde and colleagues (Serrentino et al. 2013) directly demonstrated examples of yeast hot spots with different DSB:crossover ratios. Greater propensity to form crossovers is correlated with enrichment for binding of the SUMO E3 ligase Zip3 (Serrentino et al. 2013). Although the factors that determine whether Zip3 will be enriched remain unknown, it is interesting to consider that tendency for enrichment of the Zip3 homolog RNF212 (Reynolds et al. 2013) might similarly affect the recombination outcome in mice.

The fission yeast Schizosaccharomyces pombe provides a distinct example for how recombination outcome can vary dramatically from place to place in the genome (Hyppa and Smith 2010; Fowler et al. 2014). In this organism, crossovers are distributed fairly uniformly -i.e., with nearly constant centimorgans per kilobase-despite highly nonuniform distribution of DSBs ("crossover invariance"). DSBs in hot spots tend to be repaired more often using the sister chromatid as a template, so they are less likely to give rise to interhomolog crossovers or noncrossovers. In contrast, widely dispersed DSBs that form outside of detectable hot spots are usually repaired using the homolog as a template and account for a disproportionately large fraction of crossovers. (An analogous bias toward using the sister chromatid may occur for recombination occurring near centromeres in budding yeast [Chen et al. 2008].) Available data have been interpreted to indicate that the crossover versus noncrossover ratio for interhomolog events varies little between loci in fission yeast (Cromie et al. 2005).

Because we recovered numerous noncrossovers at the distal hot spot in both males and females, it is clear that interhomolog interactions via recombination are frequent here, implying that the biases in recombination outcome predominantly reflect variation in the choice of crossover versus noncrossover pathways, not variation in the choice of homolog versus sister chromatid as the template for repair. Thus, the regional variation in recombination partner choice that underlies crossover invariance in fission yeast is distinct from the variation in crossover versus noncrossover outcome that we observed in mice.

Our findings suggest parallels with studies of recombination outcomes at a human hot spot in the PAR2 region of the $\mathrm{X}$ and $\mathrm{Y}$ chromosomes by allele-specific PCR in sperm DNA, where striking differences in crossover:noncrossover ratios were observed between men with similar or identical haplotypes within the hot spot (Sarbajna et al. 2012). This variation at a single hot spot, seen when comparing genetically distinct individuals, indicates that the 
choice of recombination outcome can be modulated by factors acting in trans or in cis but at a distance (outside the hot spot proper) (Sarbajna et al. 2012). We note that PAR2 may not be fully representative of behavior of autosomal chromosome segments: It is a small region of shared homology at the distal tips of the short arms of the $\mathrm{X}$ and $\mathrm{Y}$ chromosomes. Unlike an autosomal segment, it is not flanked by large swathes of DNA capable of pairing and recombining with the homolog, but it is also unlike the longer PAR1 region at the other end of the sex chromosomes, where crossing over occurs in nearly every meiosis and is critical for accurate sex chromosome segregation (Rouyer et al. 1986). Nonetheless, the behavior of PAR2 provides a clear example in which hot spot context (in this case, genetic and/or epigenetic differences outside the hot spot) influences the likelihood that a DSB will give rise to a crossover. In broad strokes, this appears analogous to the sex-specific difference that we observed for the distal hot spot.

We were intrigued that two of the coconversions at the distal hot spot in females had unusually long conversion tracts that spanned or flanked the region in which crossover activity in males was highest. Such long tracts have not been observed in males (Guillon et al. 2005; Svetlanov et al. 2008; Cole et al. 2010). Although the number of recovered events is small, the possibility arises that these coconversions derive from a recombination intermediate qualitatively different from most other noncrossovers. For example, these events could be explained if they started out as double Holliday junctions in the crossover pathway but then became noncrossovers by either an unusual configuration of Holliday junction resolution or Holliday junction dissolution. If so, this would imply the existence of an additional crossover control point at the double Holliday junction stage, as has been proposed in yeast (Martini et al. 2011).

\section{Sex-specific crossover activity at the distal hot spot}

In addition to differences in the crossover:noncrossover ratio between hot spots, our study also demonstrates an example of an extreme difference in the behavior of a single hot spot when assayed in the different cellular contexts of the oocyte and spermatocyte. Based on both the frequency and distribution of noncrossovers, the distal hot spot can be considered as comparably highly active in females and males, yet crossovers were observed only in males. To our knowledge, this is the first direct evidence of intrinsic bias in recombination outcome in mammalian meiosis and the first evidence in any organism that such bias can change in a cell type-specific manner. It is interesting in this regard that males have a higher likelihood of undergoing crossovers in the centromere-distal regions of chromosomes compared with females. Other crossover-suppressed hot spots may be uncovered in the distal portion of chromosomes in females. Mouse pedigree studies mapping crossovers in both male and female meioses on chromosomes 1 and 11 identified numerous hot spots. Some hot spots were sex-specific, and some of the hot spots shared between the sexes showed dif- ferent crossover frequencies in males versus females (Paigen et al. 2008; Billings et al. 2010). Thus, while the overall propensity toward crossing over is greater in males in the centromere-distal region, the crossover to noncrossover ratio will likely vary considerably between individual hot spots.

Our finding of intrinsic differences in crossover versus noncrossover frequencies both between hot spots and at the same hot spot between males and females provides new insight into the degree to which recombination outcome can be locally regulated in mammals. Although the presence of a DSB is an absolute prerequisite for crossover formation, our findings show that high DSB levels do not guarantee a high crossover activity or, as exemplified by the distal hot spot in females, any crossover activity at all. Thus, hot spot activity is regulated at multiple points from DSB formation through to the crossover/noncrossover decision.

\section{Materials and methods}

Mouse strains

The $\mathrm{A} / \mathrm{J} \times \mathrm{C} 57 \mathrm{BL} / 6 \mathrm{~J}$ F1 hybrids used in this study were either directly purchased (males) or bred from strains from the Jackson Laboratory (females and males). All experiments were done according to relevant regulatory standards and were approved by the Memorial Sloan Kettering Cancer Center Institutional Animal Care and Use Committee.

\section{DNA extraction}

DNA extractions were performed as described (Kauppi et al. 2009; Cole and Jasin 2011; de Boer et al. 2013). DNA was extracted and analyzed from two males and two pools of $\sim 45$ females. Sperm DNA was extracted from cauda epididymides from adults. Ovary DNA was extracted from newborns that were born on days 19-21 of gestation. A cell suspension was made from collected ovaries and enriched for oocytes (Eppig and Schroeder 1989; de Boer et al. 2013). A small aliquot of ovary cell suspension was used for immunocytological labeling with anti-SYCP3 to determine the fraction of oocytes (Baudat and de Massy 2007a, 2009; de Boer et al. 2009), which averaged 35\%. Liver DNA from the same mice that provided the sperm or ovary DNA served as a negative control.

\section{Hot spot identification and confirmation}

The central hot spot was identified using A/J $\times$ C57BL/6J RI strains as described (see the Supplemental Material; Bois 2007). Information about the distal hot spot was generously provided by G. Petukhova and R.D. Camerini-Otero (Smagulova et al. 2011). SNPs from Shifman et al. (2006) and the dbSNP database (NCBI) were confirmed, and additional SNPs and indels were identified by sequencing genomic DNA from both parental strains (Jackson Laboratory) (primers in Supplemental Tables S3A, S4A). Allele-specific and universal PCR primers for both hot spots were designed and optimized as described (Supplemental Tables S1, S2 [allele-specific primers], S3B, S4B [universal primers]; Kauppi et al. 2009). Amplification efficiency for each DNA sample and each primer (allele-specific and universal) was determined by performing 16 PCRs with inputs of 12,24 , and $60 \mathrm{pg}$ of DNA per reaction for each allele-specific PCR primer 
against a universal PCR primer (Cole and Jasin 2011). An initial test of hot spot activity was conducted by amplification of crossover molecules as described (Kauppi et al. 2009; Cole and Jasin 2011) with pools ranging from 300 to 3000 input DNA molecules. Liver DNA was used as a somatic (negative) control at equivalent total input DNA.

\section{Recombination assays}

For the crossover-specific assay, amplification of recombinant molecules using allele-specific primers (Fig. 1B, panel i; Supplemental Tables S1, S2) and pools of 150-400 input DNA molecules was performed as described (Kauppi et al. 2009; Cole and Jasin 2011). Crossover assays were performed in both orientations; liver DNA and no-input DNA were used as negative controls. Crossover frequencies were corrected for amplification efficiency and calculated (with estimates of standard deviation) using Poisson correction as described (Baudat and de Massy 2009), except that amplification efficiency was considered a constant for each set of primers. Crossover-positive PCRs and negative controls were reamplified using nested universal PCR primers (Supplemental Tables S3B, S4B), PCR products were transferred onto nylon membranes, and crossover breakpoints were mapped by hybridization with allele-specific oligos (Supplemental Tables S1, S2) as described (Kauppi et al. 2009).

For the noncrossover/crossover assay, recombinant molecules (along with nonrecombinant molecules of the selected haplotype) were amplified using allele-specific PCR primers against universal PCR primers with pools of 15 input DNA molecules (Fig. 1B, panel ii; Supplemental Tables S1, S2, S3B, S4B) as described (Kauppi et al. 2009; Cole and Jasin 2011). Assays were performed in all four possible primer orientations, except for the central hot spot in females. PCR products were transferred onto nylon membranes, and noncrossovers and crossovers were detected and mapped by hybridization with allele-specific oligos as described (Kauppi et al. 2009; Cole and Jasin 2011). Noncrossover and crossover frequencies were corrected for amplification efficiency and calculated using Poisson correction as described for the crossover assay. For both calculation of the overall noncrossover frequencies and the graphical representations of noncrossovers across the hot spots, coconversions were normalized by dividing by the number of polymorphisms involved.

For females, the observed crossover and noncrossover frequencies were corrected for the fraction of oocyte-derived amplifiable molecules for each ovary DNA sample as described (Baudat and de Massy 2009), except that the correction factor was considered a constant.

\section{Comparison of SSDS read counts and crossover frequencies at published mouse hot spots}

SDSS data were from Brick et al. (2012). Crossover frequencies were from Buchner et al. (2003), Yauk et al. (2003), Bois (2007), Kauppi et al. (2007), Cole et al. (2010), and Wu et al. (2010) (see also Supplemental Table S5). The crossover data used are for F1 hybrids involving the B6 strain background. With the exception of the central, distal, and $A 3$ hot spots, correction factors for amplification efficiencies were not determined separately for specific primer pairs, so crossover frequencies may be underestimated to different (unknown) degrees in data from different sources. However, from modeling of the effects of additional correction factors comparable with those that we observed in this study, it is unlikely that this uncertainty is a substantial contributor to the weakness of the regression relationship shown in Figure 3B (data not shown). For some hot spots, reciprocal crossover asymmetry has been observed, indicative of different DSB frequencies on the two haplotypes in the F1 hybrid assayed (Supplemental Table S5). For example, HS22 displayed reciprocal crossover asymmetry in the $\mathrm{B} 6 \times \mathrm{DBA}$ F1 hybrid, with the orientation of asymmetry indicating that DSB formation is more frequent on the B6 chromosome (Bois 2007). This implies in turn that relative DSB activity in the F1 hybrid is overestimated by using SSDS data from a pure B6 background. Importantly, correcting for this would not improve the overall regression relationship but would instead make it worse, so the overall poor relationship between SSDS frequency and crossover frequency is not simply a consequence of comparing data derived from different strain backgrounds.

\section{Acknowledgments}

We are grateful to Galina Petukhova and Julian Lange for sharing data prior to publication, and Liisa Kauppi and Francesca Cole for advice on performing allele-specific PCR assays for meiotic recombination. This work was supported by National Institutes of Health grant R01 HD053855 (to S.K. and M.J.). E.d.B. was supported in part by Netherlands Organization for Scientific Research Rubicon grant 825.07 .006 . S.K. is an Investigator of the Howard Hughes Medical Institute.

\section{References}

Allers T, Lichten M. 2001. Intermediates of yeast meiotic recombination contain heteroduplex DNA. Mol Cell 8: 225231.

Arbeithuber B, Betancourt AJ, Ebner T, Tiemann-Boege I. 2015. Crossovers are associated with mutation and biased gene conversion at recombination hotspots. Proc Nat1 Acad Sci 112: 2109-2114.

Baker CL, Walker M, Kajita S, Petkov PM, Paigen K. 2014. PRDM9 binding organizes hotspot nucleosomes and limits Holliday junction migration. Genome Res 24: 724-732.

Baudat F, de Massy B. 2007a. Cis- and trans-acting elements regulate the mouse Psmb9 meiotic recombination hotspot. PLoS Genet 3: e100.

Baudat F, de Massy B. 2007b. Regulating double-stranded DNA break repair towards crossover or non-crossover during mammalian meiosis. Chromosome Res 15: 565-577.

Baudat F, de Massy B. 2009. Parallel detection of crossovers and noncrossovers in mouse germ cells. Methods Mol Biol 557: 305-322.

Baudat F, Buard J, Grey C, Fledel-Alon A, Ober C, Przeworski M, Coop G, de Massy B. 2010. PRDM9 is a major determinant of meiotic recombination hotspots in humans and mice. Science 327: 836-840.

Baudat F, Imai Y, de Massy B. 2013. Meiotic recombination in mammals: localization and regulation. Nat Rev Genet 14: 794-806.

Billings T, Sargent EE, Szatkiewicz JP, Leahy N, Kwak I-Y, Bektassova N, Walker M, Hassold T, Graber JH, Broman KW, et al. 2010. Patterns of recombination activity on mouse chromosome 11 revealed by high resolution mapping. PLoS One 5: e15340.

Bois PRJ. 2007. A highly polymorphic meiotic recombination mouse hot spot exhibits incomplete repair. Mol Cell Biol 27: 7053-7062.

Brick K, Smagulova F, Khil P, Camerini-Otero RD, Petukhova GV. 2012. Genetic recombination is directed away from functional genomic elements in mice. Nature 485: 642-645. 
Buchner DA, Trudeau M, George AL Jr, Sprunger LK, Meisler MH. 2003. High-resolution mapping of the sodium channel modifier Scnm 1 on mouse chromosome 3 and identification of a 1.3-kb recombination hot spot. Genomics 82: 452-459.

Chen SY, Tsubouchi T, Rockmill B, Sandler JS, Richards DR, Vader G, Hochwagen A, Roeder GS, Fung JC. 2008. Global analysis of the meiotic crossover landscape. Dev Cell 15: 401-415.

Clement Y, Arndt PF. 2013. Meiotic recombination strongly influences GC-content evolution in short regions in the mouse genome. Mol Biol Evol 30: 2612-2618.

Cole F, Jasin M. 2011. Isolation of meiotic recombinants from mouse sperm. Methods Mol Biol 745: 251-282.

Cole F, Keeney S, Jasin M. 2010. Comprehensive, fine-scale dissection of homologous recombination outcomes at a hot spot in mouse meiosis. Mol Cell 39: 700-710.

Cole F, Baudat F, Grey C, Keeney S, de Massy B, Jasin M. 2014. Mouse tetrad analysis provides insights into recombination mechanisms and hotspot evolutionary dynamics. Nat Genet 46: $1072-1080$.

Cromie GA, Rubio CA, Hyppa RW, Smith GR. 2005. A natural meiotic DNA break site in Schizosaccharomyces pombe is a hotspot of gene conversion, highly associated with crossing over. Genetics 169: 595-605.

de Boer E, Stam P, Dietrich AJJ, Pastink A, Heyting C. 2006. Two levels of interference in mouse meiotic recombination. Proc Natl Acad Sci 103: 9607-9612.

de Boer E, Lhuissier FG, Heyting C. 2009. Cytological analysis of interference in mouse meiosis. Methods Mol Biol 558: 355-382.

de Boer E, Jasin M, Keeney S. 2013. Analysis of recombinants in female mouse meiosis. Methods Mol Biol 957: 19-45.

Dietrich AJ, Mulder RJ. 1983. A light- and electron microscopic analysis of meiotic prophase in female mice. Chromosoma 88: 377-385.

Drouaud J, Camilleri C, Bourguignon PY, Canaguier A, Berard A, Vezon D, Giancola S, Brunel D, Colot V, Prum B, et al. 2006. Variation in crossing-over rates across chromosome 4 of Arabidopsis thaliana reveals the presence of meiotic recombination 'hot spots'. Genome Res 16: 106-114.

Duret L, Galtier N. 2009. Biased gene conversion and the evolution of mammalian genomic landscapes. Annu Rev Genomics Hum Genet 10: 285-311.

Eppig JJ, Schroeder AC. 1989. Capacity of mouse oocytes from preantral follicles to undergo embryogenesis and development to live young after growth, maturation, and fertilization in vitro. Biol Reprod 41: 268-276.

Fowler KR, Sasaki M, Milman N, Keeney S, Smith GR. 2014. Evolutionarily diverse determinants of meiotic DNA break and recombination landscapes across the genome. Genome Res 24: 1650-1664.

Goldfarb T, Lichten M. 2010. Frequent and efficient use of the sister chromatid for DNA double-strand break repair during budding yeast meiosis. PLoS Biol 8: e1000520.

Grey C, Barthès P, Chauveau-Le Friec G, Langa F, Baudat F, de Massy B. 2011. Mouse PRDM9 DNA-binding specificity determines sites of histone $\mathrm{H} 3$ lysine 4 trimethylation for initiation of meiotic recombination. PLoS Biol 9: e1001176.

Guillon H, de Massy B. 2002. An initiation site for meiotic crossing-over and gene conversion in the mouse. Nat Genet 32: 296-299.

Guillon H, Baudat F, Grey C, Liskay RM, de Massy B. 2005. Crossover and noncrossover pathways in mouse meiosis. Mol Cell 20: $563-573$.
Holloway K, Lawson VE, Jeffreys AJ. 2006. Allelic recombination and de novo deletions in sperm in the human $\beta$-globin gene region. Hum Mol Genet 15: 1099-1111.

Hunter N, Kleckner N. 2001. The single-end invasion: an asymmetric intermediate at the double-strand break to double-holliday junction transition of meiotic recombination. Cell 106: 59-70.

Hyppa RW, Smith GR. 2010. Crossover invariance determined by partner choice for meiotic DNA break repair. Cell 142: 243-255.

Jeffreys AJ, May CA. 2003. DNA enrichment by allele-specific hybridization (DEASH): a novel method for haplotyping and for detecting low-frequency base substitutional variants and recombinant DNA molecules. Genome Res 13: 2316-2324.

Jeffreys AJ, May CA. 2004. Intense and highly localized gene conversion activity in human meiotic crossover hot spots. Nat Genet 36: 151-156.

Jeffreys AJ, Neumann R. 2002. Reciprocal crossover asymmetry and meiotic drive in a human recombination hot spot. Nat Genet 31: 267-271.

Jeffreys AJ, Kauppi L, Neumann R. 2001. Intensely punctate meiotic recombination in the class II region of the major histocompatibility complex. Nat Genet 29: 217-222.

Jones GH, Franklin FC. 2006. Meiotic crossing-over: obligation and interference. Cell 126: 246-248.

Kauppi L, Jasin M, Keeney S. 2007. Meiotic crossover hotspots contained in haplotype block boundaries of the mouse genome. Proc Natl Acad Sci 104: 13396-13401.

Kauppi L, May CA, Jeffreys AJ. 2009. Analysis of meiotic recombination products from human sperm. Methods Mol Biol 557: 323-355.

Lam I, Keeney S. 2014. Mechanism and regulation of meiotic recombination initiation. Cold Spring Harb Perspect Biol 7: a016634.

Libuda DE, Uzawa S, Meyer BJ, Villeneuve AM. 2013. Meiotic chromosome structures constrain and respond to designation of crossover sites. Nature 502: 703-706.

Louis EJ, Haber JE. 1990. Mitotic recombination among subtelomeric $\mathrm{Y}^{\prime}$ repeats in Saccharomyces cerevisiae. Genetics 124: 547-559.

Louis EJ, Naumova ES, Lee A, Naumov G, Haber JE. 1994. The chromosome end in yeast: its mosaic nature and influence on recombinational dynamics. Genetics 136: 789-802.

Mancera E, Bourgon R, Brozzi A, Huber W, Steinmetz LM. 2008. High-resolution mapping of meiotic crossovers and non-crossovers in yeast. Nature 454: 479-485.

Martini E, Borde V, Legendre M, Audic S, Regnault B, Soubigou G, Dujon B, Llorente B. 2011. Genome-wide analysis of heteroduplex DNA in mismatch repair-deficient yeast cells reveals novel properties of meiotic recombination pathways. PLOS Genet 7: e1002305.

McClellan KA, Gosden R, Taketo T. 2003. Continuous loss of oocytes throughout meiotic prophase in the normal mouse ovary. Dev Biol 258: 334-348.

Myers S, Bowden R, Tumian A, Bontrop RE, Freeman C, MacFie TS, McVean G, Donnelly P. 2010. Drive against hotspot motifs in primates implicates the PRDM9 gene in meiotic recombination. Science 327: 876-879.

Odenthal-Hesse L, Berg IL, Veselis A, Jeffreys AJ, May CA. 2014. Transmission distortion affecting human noncrossover but not crossover recombination: a hidden source of meiotic drive. PLoS Genet 10: e1004106.

Paigen K, Szatkiewicz JP, Sawyer K, Leahy N, Parvanov ED, Ng SHS, Graber JH, Broman KW, Petkov PM. 2008. The 
recombinational anatomy of a mouse chromosome. PLoS Genet 4: e1000119.

Pratto F, Brick K, Khil P, Smagulova F, Petukhova GV, CameriniOtero RD. 2014. DNA recombination. Recombination initiation maps of individual human genomes. Science 346: 1256442.

Reynolds A, Qiao H, Yang Y, Chen JK, Jackson N, Biswas K, Holloway JK, Baudat F, de Massy B, Wang J, et al. 2013. RNF212 is a dosage-sensitive regulator of crossing-over during mammalian meiosis. Nat Genet 45: 269-278.

Rouyer F, Simmler MC, Johnsson C, Vergnaud G, Cooke HJ, Weissenbach J. 1986. A gradient of sex linkage in the pseudoautosomal region of the human sex chromosomes. Nature 319: 291-295.

Sarbajna S, Denniff M, Jeffreys AJ, Neumann R, Soler Artigas M, Veselis A, May CA. 2012. A major recombination hotspot in the XqYq pseudoautosomal region gives new insight into processing of human gene conversion events. Hum Mol Genet 21: 2029-2038.

Schwacha A, Kleckner N. 1994. Identification of joint molecules that form frequently between homologs but rarely between sister chromatids during yeast meiosis. Cell 76: 51-63.

Serrentino ME, Chaplais E, Sommermeyer V, Borde V. 2013. Differential association of the conserved SUMO ligase Zip3 with meiotic double-strand break sites reveals regional variations in the outcome of meiotic recombination. PLoS Genet 9: e1003416.

Shifman S, Bell JT, Copley RR, Taylor MS, Williams RW, Mott R, Flint J. 2006. A high-resolution single nucleotide polymorphism genetic map of the mouse genome. PLoS Biol 4: e395.

Shiroishi T, Hanzawa N, Sagai T, Ishiura M, Gojobori T, Steinmetz M, Moriwaki K. 1990. Recombinational hotspot specific to female meiosis in the mouse major histocompatibility complex. Immunogenetics 31: 79-88.

Smagulova F, Gregoretti IV, Brick K, Khil P, Camerini-Otero RD, Petukhova GV. 2011. Genome-wide analysis reveals novel molecular features of mouse recombination hotspots. Nature 472: $375-378$.

Svetlanov A, Baudat F, Cohen PE, de Massy B. 2008. Distinct functions of MLH3 at recombination hot spots in the mouse. Genetics 178: 1937-1945.

Wu ZK, Getun IV, Bois PR. 2010. Anatomy of mouse recombination hot spots. Nucleic Acids Res 38: 2346-2354.

Yauk CL, Bois PR, Jeffreys AJ. 2003. High-resolution sperm typing of meiotic recombination in the mouse MHC E $\beta$ gene. EMBO I 22: $1389-1397$.

Youds JL, Boulton SJ. 2011. The choice in meiosis-defining the factors that influence crossover or non-crossover formation. I Cell Sci 124: 501-513. 


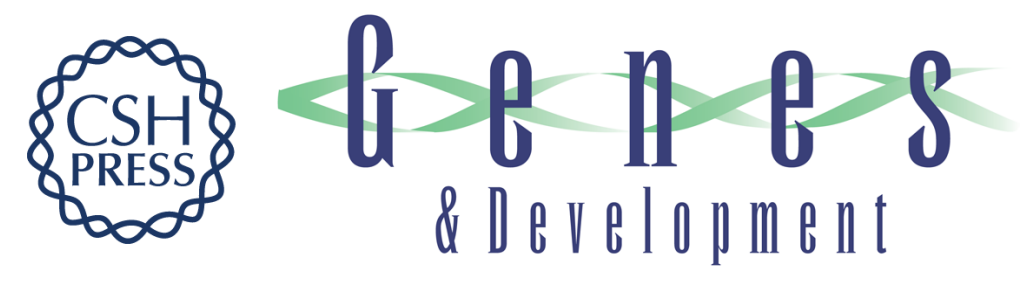

\section{Local and sex-specific biases in crossover vs. noncrossover outcomes at meiotic recombination hot spots in mice}

Esther de Boer, Maria Jasin and Scott Keeney

Genes Dev. 2015, 29: originally published online August 6, 2015

Access the most recent version at doi:10.1101/gad.265561.115

\section{Supplemental http://genesdev.cshlp.org/content/suppl/2015/08/05/gad.265561.115.DC1 Material}

References This article cites 63 articles, 17 of which can be accessed free at: http://genesdev.cshlp.org/content/29/16/1721.full.html\#ref-list-1

Creative This article is distributed exclusively by Cold Spring Harbor Laboratory Press for the first Commons six months after the full-issue publication date (see

License http://genesdev.cshlp.org/site/misc/terms.xhtml). After six months, it is available under a Creative Commons License (Attribution-NonCommercial 4.0 International), as described at http://creativecommons.org/licenses/by-nc/4.0/.

Email Alerting Receive free email alerts when new articles cite this article - sign up in the box at the top Service right corner of the article or click here.

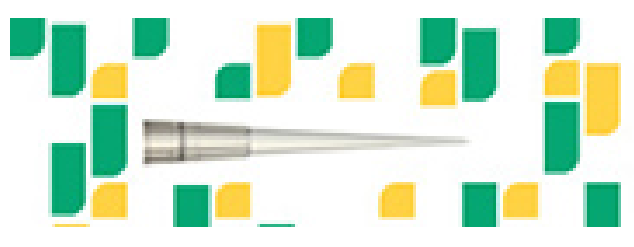

Focused on your science. 